\title{
HDCT is an effective salvage approach
}

Germ-cell tumours (GCTs) are the commonest of all tumours in men between $15-35$ years of age. Despite the majority of patients with these tumours being curable using chemotherapy, some patients will relapse and require salvage therapy. Now, a retrospective analysis of data from 364 patients with GCTs (of whom 316 had testicular GCTs) who received highdose chemotherapy (HDCT) and peripheralblood stem-cell transplantation (PBSCT) indicate that many of these patients can obtain remission with this approach, despite the failure of several prior lines of therapy.

All patients included in this analysis received HDCT plus PBSCT at Indiana University, between 2004 and 2014, having failed at least one prior line of chemotherapy. Nearly half (48\%) of patients in the cohort were initially classified as having poor-risk disease, for which cisplatin-based chemotherapy is effective in around 50\% of patients.

The majority of patients (94\%) received two courses of HDCT consisting of carboplatin $\left(700 \mathrm{mg} / \mathrm{m}^{2}\right)$ plus etoposide $\left(750 \mathrm{mg} / \mathrm{m}^{2}\right)$ on days 3, 4 and 5 after PBSCT. An estimated 2-year progression-free survival (PFS) rate of $60 \%$ was reported in patients treated with this approach, with an estimated overall survival rate of $66 \%$. Gastrointestinal toxicities were the commonest nonhaematological toxicities: grade $\geq 3$ gastrointestinal toxicities were observed in $13.8 \%$ of patients.

Remarkably, cures were achieved in patients with platinum-refractory disease, those with brain metastases, and in those who had failed to respond to three prior lines of chemotherapy, thus demonstrating the remarkable chemosensitivity of GCTs compared with that of other solid tumours.

These findings demonstrate the effectiveness of HDCT in patients with advanced-stage treatment-refractory GCTs, although further research is required to identify patients that are most likely to benefit from this approach.

Peter Sidaway

ORIGINAL ARTICLE Adra, N. et al. High-dose chemotherapy and autologous peripheral-blood stem-cell transplantation for relapsed metastatic germ cell tumors: the Indiana University experience.J. Clin. Oncol. http://dx.doi.org/10.1200/ ICO.2016.69.5395 (2016) 